(c) 2004 International Press

Adv. Theor. Math. Phys. 7 (2004) 1155-1106

\title{
Matrix Models and Gravitational \\ Corrections
}

\author{
Robbert Dijkgraaf $^{1,2}$, Annamaria Sinkovics ${ }^{1}$, and Mine \\ Temürhan ${ }^{1}$
}

${ }^{1}$ Institute for Theoretical Physics

${ }^{2}$ Korteweg-de Vries Institute for Mathematics

University of Amsterdam, 1018 XE Amsterdam, The Netherlands

\begin{abstract}
We provide evidence of the relation between supersymmetric gauge theories and matrix models beyond the planar limit. We compute gravitational $R^{2}$ couplings in gauge theories perturbatively, by summing genus one matrix model diagrams. These diagrams give the leading $1 / N^{2}$ corrections in the large $N$ limit of the matrix model and can be related to twist field correlators in a collective conformal field theory. In the case of softly broken $S U(N) \mathcal{N}=2$ super Yang-Mills theories, we find that these exact solutions of the matrix models agree with results obtained by topological field theory methods.
\end{abstract}

e-print archive: http://lanl.arXiv.org/abs/hep-th/0211241

ITFA-2002-53 


\section{Introduction}

Recently it has become clear that holomorphic or F-term information in $\mathcal{N}=1$ supersymmetric gauge theories can be exactly computed using perturbation theory, when these terms are considered as a function of the glueball superfield $S$ [1] (for earlier work see among others [2],[3],[4],[5],[6],[7]). Furthermore, for a large class of theories that allow a large $N$ expansion à la 't Hooft [8], the field theory Feynman diagrams, computed in a background super gauge field, can be seen to reduce directly to the diagrams of a zero-dimensional bosonic matrix model, where the matrix model potential is given by the gauge theory tree-level superpotential [9]; for a recent alternative derivation of this fact using anomalies see [10].

One such holomorphic quantity is the effective superpotential $W_{\text {eff }}(S)$ that is given by summing just the planar diagrams, even for a finite rank gauge theory. Non-planar diagrams will in general contribute to gravitational corrections [1]. In particular, diagrams with genus one topology, that give the leading $1 / N^{2}$ correction $\mathcal{F}_{1}$ to the matrix model free energy, contribute to an effective curvature term of the form

$$
\frac{1}{16 \pi^{2}} \int d^{4} x \mathcal{F}_{1}(S) \operatorname{Tr} R_{+} \wedge R_{+},
$$

with $R_{+}$the self-dual part of the Riemann curvature tensor. This induced gravitational correction measures the back-reaction of the field theory when it is placed in a curved background. The actual derivation along the lines of [9] of the matrix model Feynman rules, directly from the gauge theory Lagrangian in a curved superspace, will be presented elsewhere [11].

For exactly solvable matrix models the summation of the diagrams, of any fixed topology, in closed form can be done in principle, although the techniques become progressively cumbersome for high genus. One can thus try to compare these exact answers to known properties of four-dimensional supersymmetric gauge theories.

In this note we will compare the results for a single matrix model to the gravitational corrections that have been computed for topological field theories that are twisted versions of $\mathcal{N}=2$ supersymmetric Yang-Mills theories $[12],[13],[14],[15]$. These topological field theories are used to compute the Donaldson and Seiberg-Witten invariants of four-manifolds. The gauge theoretic results have been derived making use of the Seiberg-Witten solution [16] and holomorphy and duality arguments. In this paper we will demonstrate how these terms can also be computed using loop equations of matrix models. 
The genus one correction is simpler for many reasons. Being a first order correction to a semi-classical evaluation it is given by a fluctuation determinant, also in the matrix model. If the matrix model is exactly solvable, this gets often reflected in an emergent geometry, that in terms of topological string theory arises from a geometrical transition from an open string to a closed string description. General arguments tell us that such a dual geometry takes the form of a non-compact Calabi-Yau three-fold. Topological closed strings propagating on such a $\mathrm{CY}$ three-fold give rise to a genus one partition function $\mathcal{F}_{1}$ that can be expressed as a generalized Ray-Singer analytic torsion $[17]$

$$
\mathcal{F}_{1}=\sum_{p, q=0}^{3} p q(-1)^{p+q} \log \operatorname{det} \Delta_{p, q},
$$

where $\Delta_{p, q}=\left\{\bar{\partial}, \bar{\partial}^{\dagger}\right\}$ is the Laplacian acting on $(p, q)$ forms. In the simple class of matrix models that we consider in this paper the effective geometry is essentially given by an affine algebraic curve, and therefore we expect for general reasons an expression of the form

$$
\mathcal{F}_{1}=-\frac{1}{2} \log \operatorname{det} \Delta_{0}
$$

with $\Delta_{0}$ the (scalar) Laplacian on the algebraic curve acting on the collective bosonic field. We will verify this is indeed the case in some cases by explicit computation. This relation between matrix models and two-dimensional conformal collective field theory is a much more general feature, see e.g. $[18]$.

The plan of this paper is the following. In section 2 we state the precise relation between the matrix model and the gauge theory quantities relevant for gravitational corrections. In section 3 we consider gravitational couplings in $\mathcal{N}=2$ super Yang-Mills theories obtained by a topological twist, and make a comparison to the matrix model result for $S U(2)$. Then in section 4 we analyze the general matrix model answer in terms of a collective conformal field theory. We find that the genus one contribution can be described in terms of twist field correlation functions with extra dressing to match the loop equations. This will allow us to make a precise identification for general $S U(N)$ gauge group.

After this note was finished [19] appeared that discusses similar issues. 


\section{Superpotentials and gravitational couplings}

Let us first briefly review the main results of [6],[1] for the prototypical case of a $U(N)$ gauge theory coupled to single chiral matter field $\Phi$ in the adjoint representation. We start with a tree-level superpotential

$$
\int d^{4} x d^{2} \theta \operatorname{Tr} W(\Phi)
$$

where the polynomial $W$ has $n$ distinct critical points. If we consider a classical vacuum where one distributes $N_{i}$ of the eigenvalues of $\Phi$ in the $i$ th critical point of $W$, we have a classical breaking pattern

$$
U(N) \rightarrow U\left(N_{1}\right) \times \cdots \times U\left(N_{n}\right) .
$$

The strong coupling dynamics of the corresponding quantum vacuum is captured by the effective superpotential $W_{\text {eff }}\left(S_{i}\right)$ as a function of the glueball superfields

$$
S_{i}=\frac{1}{32 \pi^{2}} \operatorname{Tr}_{S U\left(N_{i}\right)} \mathcal{W}_{\alpha}^{2}
$$

According to the prescription of [1] this effective superpotential is given by

$$
W_{\mathrm{eff}}(S)=\sum_{i}\left[N_{i} \frac{\partial \mathcal{F}_{0}}{\partial S_{i}}+2 \pi \tau_{0} S_{i}\right]
$$

where $\tau_{0}$ is the bare coupling and $\mathcal{F}_{0}\left(S_{i}\right)$ is the free energy of the corresponding matrix model, obtained in a semi-classical expansion around the classical vacuum.

This matrix model takes the form of an integral over a $\widetilde{N} \times \widetilde{N}$ matrix $\Phi$ (here we carefully distinguish between $\widetilde{N}$, the rank of the matrix model, and $N$, the rank of the gauge theory)

$$
\frac{1}{\operatorname{vol} U(\widetilde{N})} \int d \Phi \exp \left[-\frac{1}{g_{s}} \operatorname{Tr} W(\Phi)\right]=\exp \left[-\sum_{g \geq 0} g_{s}^{2 g-2} \mathcal{F}_{g}\left(S_{i}\right)\right]
$$

with the identification $S_{i}=g_{s} \widetilde{N}_{i}$ in the 't Hooft limit $g_{s} \rightarrow 0, \widetilde{N}_{i} \rightarrow \infty$. More precisely, we have

$$
\mathcal{F}_{0}(S)=\frac{1}{2} S^{2} \log \left(S / \Lambda_{0}^{3}\right)+\mathcal{F}_{0}^{\text {pert }}(S)
$$

The first term gives rise to the Veneziano-Yankielowicz effective action of the pure Yang-Mills theory [20],

$$
W_{\text {eff }}(S)=N S \log \left(S / \Lambda^{3}\right)
$$


In the matrix model this contribution to (2.4) is reproduced as the large $\widetilde{N}$ volume of the unitary group [5].

The second term in (2.4) is given by a sum over planar diagrams that appear in the perturbative expansion of the matrix model. (See [21] for a careful description of this expansion around a vacuum with a spontaneous broken gauge symmetry.) A diagram with $\ell$ index loops comes with a factor of $S^{\ell}$. The actual physical values of $W_{\text {eff }}$ and the condensates $S_{i}$ in the quantum vacua are given by extremizing (2.2) with respect to the glueball fields $S_{i}$.

As we mentioned in the introduction this relation is not restricted to planar diagrams. There is an elegant interpretation of the higher genus diagrams that give the corrections $\mathcal{F}_{g}$ 's in terms of the coupling to a supergravity background [1]. In particular, the induced gravitational effective action obtained by putting the field theory on a curved space-time contains the F-term

$$
\frac{1}{16 \pi^{2}} \int d^{4} x \mathcal{F}_{1}(S) \operatorname{Tr} R_{+} \wedge R_{+} .
$$

where $R_{+}$is the self-dual part of the Riemann tensor. (There is of course a similar anti-holomorphic term $\overline{\mathcal{F}}_{1}$ multiplying $\operatorname{Tr} R_{-} \wedge R_{-}$.)

If we consider the partition function on a Euclidean four-manifold $M^{4}$, then this gravitational coupling induces a term

$$
\exp \mathcal{F}_{1}(S)\left(\frac{1}{2} \chi-\frac{3}{4} \sigma\right)
$$

with $\chi$ the Euler number and $\sigma$ the Hirzebruch signature of $M$.

Evaluating the term $\mathcal{F}_{1}(S)$ in perturbation theory, one finds that it is given exactly by the sum of diagrams with topology genus one, i.e. the diagrams that give the leading $1 / \widetilde{N}^{2}$ corrections in the large $\widetilde{N}$ limit of the matrix model. More precisely, $\mathcal{F}_{1}(S)$ is given as

$$
\mathcal{F}_{1}(S)=-\frac{1}{12} \sum_{i} \log \left(S_{i} / \Lambda_{0}^{3}\right)+\mathcal{F}_{1}^{\text {pert }}(S) .
$$

This expression is the gravitational analogue of (2.4). Assuming confinement, so that the only the field accounted for is $S$, the first term has an interpretation as an integrated form of the gravitational contribution to the $U(1)$ R-anomaly,

$$
\partial_{\mu} J_{5}^{\mu}=\frac{1}{16 \pi^{2}}\left[\frac{1}{2} \operatorname{Tr}_{U\left(N_{i}\right)} F \wedge F-\frac{1}{12} \operatorname{Tr} R \wedge R\right] .
$$




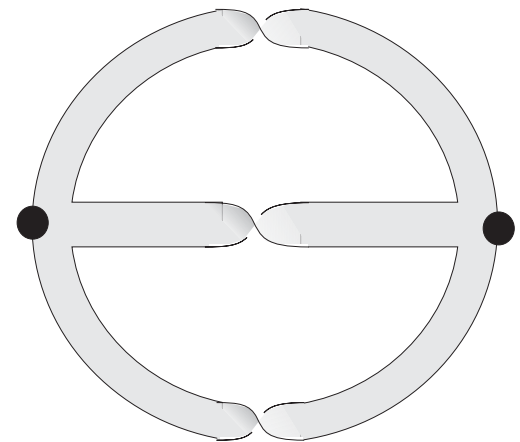

Figure 1: The simplest genus one, non-planar diagram in a cubic theory the leading perturbative contribution to $\mathcal{F}_{1}$. The $\bullet$ 's indicate insertions of the background gauge field $\mathcal{W}_{\alpha}$.

If one assumes that the low energy dynamics of the gauge system is described by an effective action in which the glueball superfields $S_{i}$ can be treated as elementary fields, the anomalous behaviour under the R-symmetry

$$
S \rightarrow e^{i \theta} S
$$

is reproduced by the combination of the Veneziano-Yankielowicz contribution $N S \log S$ to $W_{\text {eff }}(S)$ - recall that the top component of $S$ is $\operatorname{Tr} F_{+} \wedge F_{+}$ - together with the $-\frac{1}{12} \log S$ multiplying the gravitational correction. Including the complex conjugated term that muliplies $\log \bar{S}$, we see that we pick up precisely the anomaly (2.9).

The perturbative contribution $\mathcal{F}_{1}^{\text {pert }}$ to $(2.8)$ is given by summing all genus one diagrams. For example, in a cubic theory, with superpotential $W(\Phi)=m \Phi^{2}+g \Phi^{3}$ the leading diagram is given by Figure 2 and this gives

$$
\mathcal{F}_{1}^{\text {pert }}(S)=\frac{1}{2} \frac{g^{2}}{m^{3}} S+\mathcal{O}\left(S^{2}\right)
$$

Of course in the physical vacua all these expressions for $\mathcal{F}_{1}$ have to be evaluated for those values of the $S_{i}$ that minimize the effective superpotential given by the planar contribution. ${ }^{1}$

\footnotetext{
${ }^{1}$ The genus zero diagrams can also contribute, but their contribution can be shown to cancel at the critical point.
} 


\section{Matrix models and $\mathcal{N}=2$ theories}

Matrix model methods can be used in particular to find the celebrated solution to the pure $\mathcal{N}=2$ super Yang-Mills theory of Seiberg and Witten [16]. To describe the $S U(N)$ gauge theory one breaks the supersymmetry down to $\mathcal{N}=1$ by introducing a degree $N+1$ tree-level superpotential $\operatorname{Tr} W(\Phi)$ of the adjoint chiral multiplet $\Phi$ and picks the breaking pattern

$$
U(N) \rightarrow U(1)^{N}
$$

by distributing the $N$ eigenvalues of $\Phi$ equally among the $N$ critical points of $W$; that is, one chooses all $N_{i}=1$. One further decouples the diagonal $U(1)$ by putting the overall bare coupling $\tau_{0}=0$. The effective superpotential then simplifies to

$$
W_{\mathrm{eff}}(S)=\sum_{i} \frac{\partial \mathcal{F}_{0}}{\partial S_{i}}
$$

In this case the planar diagrams can be exactly summed and the solution can be written in terms of period integrals on the associated hyperelliptic Riemann surface [6]

$$
y^{2}=P(x)^{2}+f(x), \quad P(x)=W^{\prime}(x)=\sum_{i=0}^{N} u_{i} x^{N-i} .
$$

In this case the definition of the variables $S_{1}, \ldots, S_{N}$ is subtle, since they are defined in terms of the traceless piece of a $U(1)$ gauge field. Classically they vanish but as operators they make sense quantum mechanically [10]. The dependence on the $S_{i}$ is implicit in terms of the quantum deformation $f(x)$, a polynomial of degree $N-1$. After solving the constraint $d W_{\text {eff }}(S)=0$, this curve takes the familiar SW form

$$
y^{2}=P(x)^{2}-\Lambda^{2 N}
$$

By the introduction of the bare superpotential we have effectively localized to a particular point of the Coulomb branch of the $\mathcal{N}=2$ theory.

To obtain the original $\mathcal{N}=2$ model one can now scale the tree-level superpotential as $W \rightarrow \epsilon W$ and take the limit $\epsilon \rightarrow 0$. There are two obvious quantities that by a scaling argument do not depend on $\epsilon$ and can therefore be straightforwardly extracted from the $\mathcal{N}=1$ solution $^{2}$. First, there is the

\footnotetext{
${ }^{2}$ For the cubic potential $W=m \Phi^{2}+g \Phi^{3}$ this can be explicitly checked by scaling $m \rightarrow \epsilon m, g \rightarrow \epsilon g, S \rightarrow \epsilon S$. Note that in the matrix model $S=g_{s} N$ and therefore $g_{s} \rightarrow \epsilon g_{s}$.
} 
coupling matrix

$$
\tau_{i j}=\frac{\partial^{2} \mathcal{F}_{0}}{\partial S_{i} \partial S_{j}}
$$

of the $U(1)^{N}$ low-energy effective Abelian theory. Geometrically this is given by the period matrix of the curve (3.1). The second $\epsilon$-invariant quantity is the genus one free energy $\mathcal{F}_{1}$ that gives the gravitational correction (2.6).

\subsection{Gravitational coupling from topological field theory}

On flat spacetime the $\mathcal{N}=2 S U(2)$ gauge theory is described by the SeibergWitten solution. Putting the theory on a curved manifold additional gravitational terms appear in the low energy effective action. This gravitational correction has been directly computed in the $\mathcal{N}=2$ theory - more precisely, in a topological twisted version of the theory that computes Donaldson invariants. In the twisted version one modifies the action of the Lorentz group

$$
S O(4) \cong S U(2)_{+} \times S U(2)_{-} .
$$

One replaces $S U(2)_{+}$with the diagonal subgroup of $S U(2)_{+} \times S U(2)_{R}$, where the last factor is the $\mathcal{N}=2$ internal R-symmetry group [22].

In the twisted topological theory considered on a curved four-manifold $M$ these interactions are restricted to the topological $R \wedge R^{*}$ and $R \wedge R$ terms (with $R_{ \pm}=\frac{1}{2}\left(R \pm R^{*}\right)$ ) proportional to the Euler number $\chi(M)$ and the Hirzebruch signature $\sigma(M)$ respectively. The gravitational couplings contribute to the partition function with the factor

$$
\exp [b(u) \chi+c(u) \sigma]
$$

where $b(u)$ and $c(u)$ are functions of the parameter $u$ on the gauge theory moduli space.

The precise form of the functions $b(u), c(u)$ can be inferred from analyzing the modular transformation properties of the quantum theory on the curved manifold. Cancellation of the modular anomaly and additional input from the singularity structure of Seiberg-Witten moduli space determines the measure contribution [12],[13],[14],[15].

To connect these computations in topological field theory to the physical theory we recall that for manifolds with metrics of $S U(2)$ holonomy (hyperKähler manifolds) the topological twist is invisible since there is no holonomy in $S U(2)_{+}$. We can therefore directly compare to the physical gauge theory. 
In that case the metric is pure self-dual, and we have

$$
\sigma=-\frac{2}{3} \chi
$$

For example one could take $M=K 3$ for which $\chi=24$ and $\sigma=-16$. So the overall contribution to the path-integral is

$$
\exp \left[\left(b(u)-\frac{2}{3} c(u)\right) \chi\right]
$$

If we compare this to (2.7), where we use that for a self-dual geometry $\frac{1}{2} \chi-\frac{3}{4} \sigma=\chi$, we have the following identification between the matrix model and gauge theory quantities

$$
\mathcal{F}_{1}(S)=b(u)-\frac{2}{3} c(u)
$$

We will now check this relation in a number of cases. We will for convenience put $\chi=1$.

\subsection{The $\mathcal{N}=2 S U(2)$ theory}

In this case the Seiberg-Witten geometry can be described by deforming the $\mathcal{N}=2$ theory with a tree level superpotential,

$$
W^{\prime}(\Phi)=\epsilon\left(\Phi^{2}-u\right)
$$

(For more details about the perturbative derivation of this particular case see [21].) As described above, extremization of the effective glueball superpotential gives the Seiberg-Witten curve for $S U(2)$

$$
y^{2}=\left(x^{2}-u\right)^{2}-1
$$

Here the scale $\Lambda$ is set to one for convenience and the factor $\epsilon$ is absorbed. As we mentioned the physical quantities $\mathcal{F}_{1}$ and the coupling matrix $\tau_{i j}$ are independent of the deformation parameter $\epsilon$. The curve has four branch points at

$$
x_{i}= \pm \sqrt{u \pm 1} .
$$

It is described by the two-cut solution of the matrix model with the potential $W(\Phi)$ given by (3.3).

The genus one free energy for two-cut solutions in matrix models have been explicitly computed. Here we use the relevant solution of Akemann 
[23], which is an elaboration of the methods of [24],

$$
\begin{aligned}
\mathcal{F}_{1}=-\frac{1}{24} \sum_{i=1}^{4} \log M_{i}-\frac{1}{2} \log |K(k)| \\
\quad-\frac{1}{12} \Delta+\frac{1}{4} \log \left|\left(x_{1}-x_{3}\right)\left(x_{2}-x_{4}\right)\right| .
\end{aligned}
$$

This solution was derived by an iterative genus expansion of the loop equation; we discuss this further in the next section. Here $\Delta$ is the discriminant of the elliptic curve (3.4)

$$
\Delta=\prod_{i<j}\left(x_{i}-x_{j}\right)^{2}=64\left(u^{2}-1\right),
$$

and $K(k)$ is the complete elliptic integral, where the nome $k$ is expressed in the modulus $\tau$ of the SW curve. The solution also depends on the first moments of the potential that are generally defined as

$$
M_{i}=\frac{1}{2 \pi i} \oint_{C_{\infty}} d x \frac{W^{\prime}(x)}{\left(x-x_{i}\right) \sqrt{\prod_{i=1}^{4}\left(x-x_{i}\right)}} .
$$

For the simple potential (3.3) the contour can be deformed to infinity, and one gets $M_{i}=\epsilon$.

For comparison with the gauge theory result it is useful to express $\mathcal{F}_{1}$ in terms of the parameter of $S U(2)$ moduli space $u$. The elliptic parametrization of the SW curve (3.4) can be written in terms of Jacobi $\theta$ functions as

$$
u=\frac{\theta_{2}^{4}+\theta_{3}^{4}}{2\left(\theta_{2} \theta_{3}\right)^{2}}, \quad u^{2}-1=\frac{\theta_{4}^{8}}{4\left(\theta_{2} \theta_{3}\right)^{4}},
$$

where the definition of the $\theta$ functions is as usual

$$
\begin{aligned}
& \theta_{2}=\sum_{n \in \mathbf{Z}} q^{\frac{1}{2}\left(n+\frac{1}{2}\right)^{2}} \\
& \theta_{3}=\sum_{n \in \mathbf{Z}} q^{\frac{1}{2} n^{2}} \theta_{4}=\sum_{b \in \mathbf{Z}}(-1)^{n} q^{\frac{1}{2} n^{2}}
\end{aligned}
$$

with $q=e^{2 \pi i \tau}$. A useful identity they satisfy is $\theta_{2}^{4}+\theta_{4}^{4}=\theta_{3}^{4}$. The complete elliptic integral $K(k)$ can also be expressed in $\theta$ functions as

$$
K(k)=\frac{\pi}{2} \theta_{3}^{2} .
$$

With these elliptic parametrization the matrix model answer for the two-cut solution to $\mathcal{F}_{1}$ can be written as

$$
\mathcal{F}_{1}=-\frac{1}{6} \log \epsilon+\frac{1}{4} \log \frac{4}{\pi\left(\theta_{2} \theta_{3}\right)^{2}}-\frac{1}{12} \log \frac{16 \theta_{4}^{8}}{\left(\theta_{2} \theta_{3}\right)^{4}} .
$$

The factor $\log \epsilon$ can be absorbed in the measure. 


\subsection{Comparison to the gauge theory}

We now have to compare this result to the topological field theory answer that reads $[12],[13]$

$$
\begin{aligned}
& e^{b(u)}=\alpha\left(\left(u^{2}-1\right) \frac{d \tau}{d u}\right)^{1 / 4} \\
& e^{c(u)}=\beta\left(u^{2}-1\right)^{1 / 8}
\end{aligned}
$$

where $\alpha$ and $\beta$ are constant coefficients. This contribution to the partition function should match with the matrix theory computation for the corresponding genus one contribution. To check this, it helps to rewrite the gauge theory contribution as

$$
Z_{\text {gauge }}=e^{b(u)-\frac{2}{3} c(u)}=A^{-1 / 2} \Delta^{-1 / 12},
$$

with

$$
A=\frac{d a}{d u}, \quad \Delta=64\left(u^{2}-1\right)
$$

Here

$$
\left(u^{2}-1\right) \frac{d \tau}{d u}=\frac{i}{4 \pi}\left(\frac{d u}{d a}\right)^{2}
$$

is rewritten in terms of the "electric" period of the Seiberg-Witten curve a. Substituting the modular parametrization of the curve in terms of $\theta$ functions we find

$$
\Delta=\frac{16 \theta_{4}^{8}}{\left(\theta_{2} \theta_{3}\right)^{4}}, \quad A=\frac{d a}{d u}=\frac{1}{2} \theta_{2} \theta_{3},
$$

Comparing with the matrix theory contribution (3.9) we find perfect agreement.

\section{4 $S U(N)$ generalization}

The gauge theory computation for the partition function can be generalized for the $S U(N)$ theory. The generalization is based on a similar analysis of anomalies as for the $S U(2)$ case.

At a generic point on the Coulomb branch, where the gauge symmetry is broken to $U(1)^{N-1}$, the $S U(N)$ theory can be described by the hyperelliptic curve

$$
y^{2}=P(x)^{2}-1=\prod_{i=1}^{2 N}\left(x-x_{i}\right), P(x)=\sum_{i=0}^{N} u_{i} x^{N-i}, \quad \sum_{i=1}^{N} u_{i}=0 .
$$


Here the $u_{i}$ 's are the symmetric polynomials of the roots of $P(x)$, and $x_{i}$ are the branch points of the curve. The hyperelliptic curve is a Riemann surface of genus $g=N-1$.

For a genus $g$ Riemann surface one takes a basis of $2 g$ homology cycles $\left(A_{i}, B_{i}\right)$ with canonical intersection product. The periods of the curve are then related to a set of dual holomorphic one-forms $\omega_{i}=x^{i-1} d x / y$ as (we choose the homology basis slightly different then in [14],[15] in order to make contact with the matrix model basis)

$$
A_{i j}=\oint_{A_{i}} \omega_{j}=\frac{\partial a_{i}}{\partial u_{j+1}}, \quad B_{i j}=\oint_{B_{i}} \omega_{j}=\frac{\partial a_{D, i}}{\partial u_{j+1}} .
$$

The period (or coupling) matrix $\tau_{i j}$ is given as

$$
\tau_{i j}=\frac{\partial a_{D, i}}{\partial a_{j}}=\left(B A^{-1}\right)_{i j}
$$

The partition function for $S U(N)$ is a direct generalization of the corresponding $S U(2)$ contribution [14],[15]. We will write it as (discarding overall constants)

$$
Z_{\text {gauge }}=A^{-\chi / 2} \Delta^{\sigma / 8}
$$

with

$$
A=\operatorname{det} A_{i j}, \quad \Delta=\prod_{i<j}^{2 N}\left(x_{i}-x_{j}\right)^{2} .
$$

Putting a self-dual metric and $\chi=1$ we get

$$
Z_{\text {gauge }}=A^{-1 / 2} \Delta^{-1 / 12} \text {. }
$$

To compare this result to the genus one free energy of the matrix model we first have to explain how matrix model results can be computed using conformal field theory.

\section{Multicut solutions and conformal field theory}

\subsection{Loop equations and Virasoro constraints}

For the one-loop free-energy for the $S U(N)$ theory we have to solve the corresponding matrix model with the tree-level superpotential $W(\Phi)$ with $W^{\prime}(\Phi)=\epsilon P(\Phi)$ and with the maximum number of cuts. The most efficient 
way to derive multicut solutions for matrix models is by using loop equations and conformal field theory techniques. The method of using loop equations to obtain the $1 / N$ corrections in matrix models was developed in [24]. Here we will follow closely [18] that gives a good general exposition of the relation of these methods to conformal field theory.

We start from the partition function of the associated matrix model

$$
Z=\frac{1}{\operatorname{vol} U(\widetilde{N})} \int d \Phi \exp \left[-\frac{1}{g_{s}} \operatorname{Tr} W(\Phi)\right],
$$

for the $\widetilde{N} \times \widetilde{N}$ matrix $\Phi$ with a general potential $W(\Phi)$. The reparametrization invariance of this integral leads directly to the so-called loop equation ${ }^{3}$. The simplest way to derive the loop equation is taking a shift $\Phi \rightarrow \frac{\delta}{(x-\Phi)}$ where $\delta$ is a small number. This gives the equation for the loop correlator

$$
\left\langle\omega(x)^{2}-\frac{1}{g_{s}} \operatorname{Tr}\left(\frac{W^{\prime}(\Phi)}{x-\Phi}\right)\right\rangle=0,
$$

where

$$
\omega(z)=\operatorname{Tr} \frac{1}{x-\Phi}=\sum_{I=1}^{\widetilde{N}} \frac{1}{x-\lambda_{I}}
$$

is the loop operator and $\lambda_{I}$ the eigenvalues of the matrix $\Phi$. For a general potential with coupling constants $t_{n}$

$$
W(\Phi)=-\sum_{n=1}^{\infty} t_{n} \Phi^{n},
$$

the loop equation can be rewritten as

$$
\oint \frac{d x^{\prime}}{2 \pi i} \frac{1}{x-x^{\prime}}\left\langle T\left(x^{\prime}\right)\right\rangle=0
$$

where the contour includes all eigenvalues $\lambda_{I}$ but excludes the point $x$. Here we introduce the stress-tensor $T(x)$ of the collective field $\varphi(x)$

$$
T(x)=\frac{1}{2}(\partial \varphi(x))^{2}, \varphi(x)=W(x)-2 g_{s} \operatorname{Tr} \log \left(\frac{1}{x-\Phi}\right) .
$$

The loop equation can be reformulated as the Virasoro constraints [25],[26]

$$
\begin{aligned}
L_{n} Z & =0, \quad n \geq-1, \\
L_{n} & =\sum_{k=0}^{n} \frac{\partial}{\partial t_{k}} \frac{\partial}{\partial t_{n-k}}+\sum_{k=0}^{\infty} k t_{k} \frac{\partial}{\partial t_{n+k}}
\end{aligned}
$$

\footnotetext{
${ }^{3}$ These loop equations have been recently given a gauge theoretic interpretation in [10].
} 
where the operators $L_{n}$ satisfy (half of) the Virasoro algebra

$$
\left[L_{n}, L_{m}\right]=(m-n) L_{m+n} .
$$

The loop equation can be solved iteratively order by order making a $1 / \tilde{N}$ expansion. The planar limit is usually not so hard to solve, but for the next order solution one needs special techniques.

\subsection{Planar solution and effective geometry}

To solve the loop equation in the $\widetilde{N} \rightarrow \infty$ limit, it is simplest to rewrite it in terms of the polynomial

$$
f(x)=4 g_{s}\left\langle\operatorname{Tr} \frac{W^{\prime}(\Phi)-W^{\prime}(x)}{\Phi-x}\right\rangle
$$

If $W(x)$ is of degree $n+1$ then the polynomial $f(x)$ is of degree $n-1$. Denoting the classical average of the loop operator as

$$
\omega_{c}(x)=\frac{1}{\widetilde{N}}\langle\omega(x)\rangle,
$$

in the large $\tilde{N}$ limit the loop equation becomes quadratic

$$
\omega_{c}(x)^{2}-\frac{1}{g_{s} \widetilde{N}} W^{\prime}(x) \omega_{c}(x)+\frac{1}{4 g_{s}^{2} \widetilde{N}^{2}} f(x)=0 .
$$

In terms of the bosonic collective field $\varphi(x)$ we can say that this obtains a large vacuum expectation value in the planar limit, which is the solution for the classical Virasoro constraint. The current $\partial \varphi(x)$ takes its classical value

$$
\begin{aligned}
\partial \varphi_{c}(x) & =W^{\prime}(x)-2 g_{s} \widetilde{N} \omega_{c}(x) \\
= & W^{\prime}(x)-\frac{2 g_{s} \widetilde{N}}{x}+\mathcal{O}\left(\frac{1}{x^{2}}\right) .
\end{aligned}
$$

For our solution we have with $y=\partial \varphi_{c}(x)$

$$
y^{2}=W^{\prime}(x)^{2}+f(x) .
$$

We can write this as the hyperelliptic curve

$$
y^{2}=\prod_{i=1}^{2 N}\left(x-x_{i}\right)
$$

Since the classical field $\varphi(x)$ changes sign around the branch points, the expectation values of the bosonic field are given by two branches of $y(x)$. 
Then $\varphi(x)$ can be thought of as a single bosonic field defined on the branched covering given by the hyperelliptic curve (4.1). The classical value of the collective field is given as

$$
\partial \varphi_{c}(x)=\prod_{i}\left(x-x_{i}\right)^{1 / 2}
$$

\subsection{Subleading corrections and twist fields}

The subleading term $\mathcal{F}_{1}$ in the free energy is given by the Gaussian fluctuations around the classical solution. As we mentioned in the introduction, by general arguments this term is equal to $-\frac{1}{2} \log \operatorname{det} \Delta_{0}$, the Laplace operator on the Riemann surface, and additional (dressing) terms arising from the fluctuation of the branch points.

Instead of thinking of $\varphi(x)$ as a field living on the hyperelliptic Riemann surface (4.1), we can also think of it on the complex $x$-plane in the presence of twist operators $\sigma\left(x_{i}\right)$ associated with the branch points $x_{i}$. In the neighbourhood of such a twist field the current $\partial \varphi(x)$ is no longer single-valued but has a branch cut in its operator product

$$
\partial \varphi(x) \cdot \sigma\left(x_{i}\right) \sim\left(x-x_{i}\right)^{-1 / 2} \tau\left(x_{i}\right) .
$$

Here $\sigma(x)$ and $\tau(x)$ are conformal fields of dimension 1/16 and 9/16 respectively. A naive expression for the genus one contribution to the matrix model would now be given by

$$
Z_{\mathrm{twist}}=\left\langle\prod_{i=1}^{2 N} \sigma\left(x_{i}\right)\right\rangle .
$$

The chiral ${ }^{4}$ twist field correlation function is well-known [27], [28],,[29]

$$
Z_{\mathrm{twist}}=A^{-\frac{1}{2}} \prod_{i<j}\left(x_{i}-x_{j}\right)^{-1 / 8}
$$

Here

$$
A=\operatorname{det}\left(A_{i j}\right)
$$

is the determinant of the period matrix, related to the integral of the oneforms $\omega_{i}$ over the $A$-cycles

$$
A_{i j}=\oint_{A_{i}} \frac{x^{j-1} d x}{y}
$$

\footnotetext{
${ }^{4}$ In this expression the chiral projection is done by putting the loop momenta of the field $\varphi(x)$ to zero.
} 
For example, in the case of a two-point function we get the familiar result

$$
\left\langle\sigma\left(x_{1}\right) \sigma\left(x_{2}\right)\right\rangle=\left(x_{1}-x_{2}\right)^{-1 / 8}
$$

expressing the fact that the conformal dimension of a $\mathbf{Z}_{2}$ twist field is $1 / 16$.

Formula (4.3) also can be expressed as the chiral determinant of the Laplace operator $\Delta_{0}$ of the twisted boson on the hyperelliptic curve

$$
Z_{\text {twist }}=\left(\operatorname{det} \Delta_{0}\right)^{-1 / 2} \text {. }
$$

\subsection{Star operators}

However, (4.3) is not the full answer, since as it stands this expression does not solve the Virasoro constraints. An elegant solution to this has been given by Kostov in terms of star operators [18].

We can associate a Hilbert space with the local complex variable near each branch point and solve the Virasoro constraint in the vicinity of the branch point. We have to look for an operator which creates a conformally invariant state near the branch point. The twist operator itself does not satisfy all the Virasoro constraints, in particular it does not satisfy $L_{-1}$. Therefore we will look for a new operator which satisfies all constraints. Such operators are called star operators [30], and they are constructed from the modes of the twisted bosonic field near the branch point ${ }^{5}$.

The twisted bosonic current near the branch point $x_{i}$ is now decomposed into a classical and quantum part

$$
\partial \varphi(x)=\partial \varphi_{c}(x)+\sum_{r \in \mathbf{Z}+\frac{1}{2}} \alpha_{r}\left(x-x_{i}\right)^{-r-1} .
$$

The expansion of the classical current (4.2) is

$$
\partial \varphi_{c}(x)=\sum_{r \geq \frac{1}{2}} \mu_{r}\left(x_{k}\right) \cdot\left(x-x_{k}\right)^{r-1} .
$$

This defines the coefficients $\mu_{r}\left(x_{i}\right)$. The Fock vacuum for such a twist field is defined as

$$
\left|0_{i}\right\rangle=\sigma\left(x_{i}\right)|0\rangle
$$

and it satisfies

$$
\alpha_{r}\left|0_{i}\right\rangle=0, \quad r>0
$$

\footnotetext{
${ }^{5}$ We would like to thank I. Kostov for sharing with us some unpublished work on the construction of the star operators.
} 
Since it depends on the position of the branch points it is not translationally invariant. To make it invariant, one introduces the star operator

$$
S\left(x_{i}\right)=e^{s\left(x_{i}\right)} \sigma\left(x_{i}\right)
$$

and assume it is defined perturbatively by a mode expansion

$$
s\left(x_{i}\right)=\sum_{n \geq 0} \frac{1}{n !} \sum_{r_{1} \ldots r_{n}} s_{r_{1} \ldots r_{n}}\left(x_{i}\right) \alpha_{-r_{1}} \ldots \alpha_{-r_{n}} .
$$

The coefficients in the mode expansion are determined by imposing the conditions of conformal invariance

$$
L_{n} e^{s\left(x_{i}\right)}\left|0_{i}\right\rangle=0, \quad n \geq-1 .
$$

Up to $1 / \widetilde{N}^{2}$ correction one finds simply an extra multiplicative factor [18]

$$
S\left(x_{i}\right)=\left[\mu_{3 / 2}\left(x_{i}\right)\right]^{-1 / 24} \sigma\left(x_{i}\right) .
$$

The full genus one contribution to the free energy, obtained by solving the loop equation including the order $1 / \widetilde{N}^{2}$ corrections, is therefore given by the correlation function of star operators, not the twist operators,

$$
\begin{aligned}
\mathcal{F}_{1} & =\log \left\langle\prod_{i=1}^{2 N} S\left(a_{i}\right)\right\rangle \\
& =-\frac{1}{24} \sum_{i=1}^{2 N} \log \mu_{3 / 2}\left(x_{i}\right)+\log Z_{\text {twist }},
\end{aligned}
$$

where $Z_{\text {twist }}$ is the correlation function of the $2 N$ twist fields (4.3). From the expansion of the classical current $\partial \varphi_{c}(z)$ we get

$$
-\frac{1}{24} \sum_{i=1}^{2 N} \log \mu_{3 / 2}\left(x_{i}\right)=-\frac{1}{24} \log \prod_{i<j}\left(x_{i}-x_{j}\right)
$$

So the final result for $Z_{\text {matrix }}$ is then ${ }^{6}$

$$
Z_{\text {matrix }}=e^{\mathcal{F}_{1}}=A^{-1 / 2} \Delta^{-1 / 12}
$$

This result is in complete agreement with the gauge theory partition function $Z_{\text {gauge }}(3.13)$.

\section{Acknowledgements}

We would like to thank I. Kostov and C. Vafa for enlightening discussions. This research is supported by NWO, the FOM Programme String Theory and Quantum Gravity, and the CMPA grant of the University of Amsterdam.

\footnotetext{
${ }^{6}$ Note that this form is given incorrectly in [18].
} 


\section{References}

[1] R. Dijkgraaf, C. Vafa, "A Perturbative Window into Non-Perturbative Physics," arXiv:hep-th/0208048.

[2] R. Gopakumar and C. Vafa, "On the gauge theory/geometry correspondence," Adv. Theor. Math. Phys. 3, 1415 (1999) [arXiv:hepth/9811131].

[3] C. Vafa, "Superstrings and topological strings at large $N$," J. Math. Phys. 42, 2798 (2001) [arXiv:hep-th/0008142].

[4] F. Cachazo, K. A. Intriligator and C. Vafa, "A large $N$ duality via a geometric transition," Nucl. Phys. B 603, 3 (2001) [arXiv:hep-th/0103067].

[5] H. Ooguri and C. Vafa, "Worldsheet derivation of a large $N$ duality," Nucl. Phys. B 641 (2002) 3, [arXiv:hep-th/0205297].

[6] R. Dijkgraaf and C. Vafa, "Matrix models, topological strings, and supersymmetric gauge theories," arXiv:hep-th/0206255.

[7] R. Dijkgraaf and C. Vafa, "On geometry and matrix models," arXiv:hep-th/0207106.

[8] G. 't Hooft, "A Planar Diagram Theory For Strong Interactions," Nucl. Phys. B 72, 461 (1974).

[9] R. Dijkgraaf, M.T. Grisaru, C.S. Lam, C. Vafa, and D. Zanon, "Perturbative Computation of Glueball Superpotentials," [arXiv:hepth/0211017].

[10] F. Cachazo, M.R. Douglas, N. Seiberg, and E. Witten, "Chiral Rings and Anomalies in Supersymmetric Gauge Theory," JHEP 0212 (2002) 071 [arXiv:hep-th/0211170].

[11] R. Dijkgraaf, M.T. Grisaru, C.S. Lam, C. Vafa, and D. Zanon, "Planar gravitational corrections for supersymmetric gauge theories," [arXiv:hep-th/0310061].

[12] E. Witten, "On S duality in Abelian gauge theory," Selecta Math. 1 (1995) 383 arXiv:hep-th/9505186

[13] G. W. Moore and E. Witten, "Integration over the u-plane in Donaldson theory," Adv. Theor. Math. Phys. 1, 298 (1998) [arXiv:hep-th/9709193].

[14] A. Losev, N. Nekrasov and S. L. Shatashvili, "Issues in topological gauge theory," Nucl. Phys. B 534, 549 (1998) [arXiv:hep-th/9711108]. 
[15] M. Marino and G. W. Moore, "Integrating over the Coulomb branch in $N=2$ gauge theory," Nucl. Phys. Proc. Suppl. 68, 336 (1998) [arXiv:hep-th/9712062]; "The Donaldson-Witten function for gauge groups of rank larger than one," Commun. Math. Phys. 199, 25 (1998) [arXiv:hep-th/9802185]; "Donaldson invariants for non-simply connected manifolds," [arXiv:hep-th/9804104].

[16] N. Seiberg and E. Witten, "Electric-magnetic duality, monopole condensation, and confinement in $N=2$ supersymmetric Yang-Mills theory," Nucl. Phys. B 426, 19 (1994) [Erratum-ibid. B 430, 485 (1994)] [arXiv:hep-th/9407087].

[17] M. Bershadsky, S. Cecotti, H. Ooguri and C. Vafa, "Kodaira-Spencer theory of gravity and exact results for quantum string amplitudes," Commun. Math. Phys. 165, 311 (1994) [arXiv:hep-th/9309140].

[18] I. K. Kostov, "Conformal field theory techniques in random matrix models," arXiv:hep-th/9907060.

[19] A. Klemm, M. Marino, and S. Theisen, " Gravitational corrections in supersymmetric gauge theory and matrix models," JHEP 0303 (2003) 051 [arXiv:hep-th/0211216].

[20] G. Veneziano and S. Yankielowicz, "An Effective Lagrangian For The Pure $N=1$ Supersymmetric Yang-Mills Theory," Phys. Lett. B 113, 231 (1982).

[21] R. Dijkgraaf, S. Gukov, V.A. Kazakov, C. Vafa, "Perturbative Analysis of Gauged Matrix Models," Phys. Rev. D 68 (2003) 045007 [arXiv:hepth/0210238].

[22] E. Witten, "Topological quantum field theory," Commun. Math. Phys. 117 (1988) 353.

[23] G. Akemann, "Higher genus correlators for the Hermitian matrix model with multiple cuts," Nucl. Phys. B 482, 403 (1996) [arXiv:hepth/9606004].

[24] J. Ambjorn, L. Chekhov, C. F. Kristjansen and Y. Makeenko, "Matrix model calculations beyond the spherical limit," Nucl. Phys. B 404, 127 (1993) [Erratum-ibid. B 449, 681 (1995)] [arXiv:hep-th/9302014].

[25] R. Dijkgraaf, H. Verlinde and E. Verlinde, "Loop Equations And Virasoro Constraints In Nonperturbative 2-D Quantum Gravity," Nucl. Phys. B 348, 435 (1991). 
[26] M. Fukuma, H. Kawai and R. Nakayama, "Continuum Schwinger-Dyson Equations And Universal Structures In Two-Dimensional Quantum Gravity," Int. J. Mod. Phys. A 6, 1385 (1991).

[27] L. J. Dixon, D. Friedan, E. J. Martinec and S. H. Shenker, "The Conformal Field Theory Of Orbifolds," Nucl. Phys. B 282, 13 (1987).

[28] A. B. Zamolodchikov, "Conformal Scalar Field On The Hyperelliptic Curve And Critical Ashkin-Teller Multipoint Correlation Functions," Nucl. Phys. B 285, 481 (1987).

[29] M. A. Bershadsky and A. O. Radul, "Conformal Field Theories With Additional $Z(N)$ Symmetry," Sov. J. Nucl. Phys. 47, 363 (1988) [Yad. Fiz. 47, 575 (1988)].

[30] G. W. Moore, "Matrix Models Of 2-D Gravity And Isomonodromic Deformation," lectures at 1990 Cargese Workshop on Random Surfaces, Quantum Gravity and Strings, Prog. Theor. Phys. Suppl. 102, 255 (1990). 